\title{
Time-restricted feeding increases fat oxidation rate but not affect postprandial lipemia: a crossover trial
}

Chih-Hui Chiu ( $\nabla$ chiuch@ntus.edu.tw)

National Taiwan University of Sport

Che-Hsiu Chen

National Taiwan University of Sport

Min-Huan Wu

Tunghai University

\section{Yu-Chen Hsieh}

National Taiwan University of Sport

Zong-Yan Lin

National Taiwan University of Sport

Bo-Wei Chen

National Taiwan University of Sport

\section{Research Article}

Keywords: intermittent fasting, oral fat tolerance test, fat oxidation

Posted Date: January 20th, 2022

DOI: https://doi.org/10.21203/rs.3.rs-1267469/v1

License: (c) (i) This work is licensed under a Creative Commons Attribution 4.0 International License.

Read Full License 


\section{Abstract}

Background: Studies have revealed that time-restricted feeding affects the fat oxidation rate; however, its effects on the fat oxidation rate and hyperlipidemia following high-fat meals are unclear. This study investigated the effects of 5-day time-restricted feeding on the fat oxidation rate and postprandial lipemia following high fat meals.

Methods: In this random crossover experimental study, eight healthy male adults were included each in the 5-day time-restricted feeding trial and the control trial. The meals of the time-restricted feeding trial were provided at 12:00,16:00, and 20:00. The meals of the control trial were provided at 08:00, 14:00, and 20:00. The contents of the meals of both trials were the same, and the calories of the meals met the 24hour energy requirement of the participants. After 5 days of the intervention, the participants consumed high-fat meals on the sixth day, and their physiological changes were determined.

Results: The fasting fat oxidation rate $(p<0.001)$ and postprandial fat oxidation rate $(p=0.019)$ of the time-restricted feeding trial were significantly higher than those of the control trial. The 24-hour energy consumption and postprandial triglyceride, blood glucose, insulin, glycerol, and free fatty acid concentrations of the two trials showed no significant differences $(p>0.05)$.

Conclusion: The results revealed that 5 days of time-restricted feeding effectively increased the fasting and postprandial fat oxidation rate, but it did not affect postprandial lipemia.

\section{Introduction}

Consuming high-fat meals increases the triglyceride (TG) level in blood plasma. Studies have discovered that large increases in postprandial TG concentration lead to high risks of cardiovascular diseases and metabolic syndrome (1). Compared with the fasting TG concentration, the postprandial TG concentration is a more precise predictor of the risks of cardiovascular diseases and metabolic syndrome (2). Consuming high-fat meals increases the levels of biochemical substances in blood plasma, such as TG, free fatty acids, and remnant cholesterol. Studies have reported that these biochemical substances are major risk factors for metabolic syndrome, atherosclerosis, myocardial infarction, and coronary heart disease, all of which are associated with high mortality $(3,4)$. The high TG level after the consumption of high-fat meals can last for 6-8 hours. As three meals daily are typically consumed by the general population, high levels of TG may be constantly occurring in the body. Therefore, investigating methods to reduce the high TG level after eating high-fat meals is crucial for reducing the development of metabolic syndrome.

Studies have demonstrated that a single session of endurance exercise can effectively reduce the TG level after the consumption of high-fat meals (5-8). In addition, performing a single session of endurance exercise 8 hours before meals can effectively reduce the increase in TG after the consumption of high-fat meals, and the effect can last for 48-60 hours $(9,10)$. Endurance exercises effectively reduce the increase in postprandial TG because they increase lipoprotein lipase activity and insulin sensitivity, and 
they reduce the release of very low-density lipoprotein by the liver (10). Recent studies have shown that increasing the fat oxidation rate after eating high-fat meals is crucial for reducing the postprandial TG level $(11,12)$; however, the results have remained inconsistent. Some studies have described that performing high-intensity interval training is positively correlated with decreases in the postprandial TG level (13), whereas other studies have discovered that the increased fat oxidation rate following high-fat meals did not affect the postprandial TG level (14).

Studies have discovered that time-restricted feeding can improve insulin sensitivity (15), increase the fat oxidation rate (16), and decrease the fasting TG level (17). However, whether time-restricted feeding can exert health benefits in terms of effectively reducing the increase in the TG level following high-fat meals remains unclear. This study investigated the effects of 5-day time-restricted feeding on the fat oxidation rate and postprandial lipemia after the consumption of high-fat meals.

\section{Methods}

\section{Participants}

This study recruited eight healthy male adults as research participants (age: $22 \pm 1.3 \mathrm{yr}$, height: $170.1 \pm$ $4.7 \mathrm{~cm}$, weight: $75.4 \pm 17.5 \mathrm{~kg}$ ). All the participants had not undergone physical training; they did not exercise regularly; and they did not have any diseases that would prevent them from performing exercises, such as high blood pressure, hyperlipidemia, heart disease, joint disease, and osteoporosis. All the participants fully understood the experimental process before experiment initiation and were notified of the possible risks; they agreed to the terms of the experiment and provided their written consent. A similar number of participants and a similar recruitment method have been employed by this research team in the past. This study was approved by the Institutional Review Board of Jen-Ai Hospital (110-10) in Taiwan.

\section{Design}

This study used a crossover design for the experiment. The participants were divided into the timerestricted feeding trial and the control trial; the participants in the control trial did not practice intermittent fasting methods. The participants of both trials consumed the same meals for 5 days. The time-restricted feeding group used the 16:8 methods to practice intermittent fasting. The meals were provided at 12:00, 16:00, and 20:00. The meals of the control group were provided at 08:00, 14:00, and 20:00, but the consumption time was not limited. On the morning of the sixth day, all participants returned to the laboratory to consume high-fat meals, and their TG blood levels after the meal was determined. The participants were randomly assigned to different arms of the study to receive different treatments, and an interval of at least 14 days was maintained between the tests to avoid any effects of the preceding test on the succeeding test. Studies have discovered that 4 days of intermittent fasting effectively increased the fat oxidation rate and reduced blood glucose $(18,19)$. Therefore, 5 days of intermittent fasting should provide sufficient intervention time to stimulate fat oxidation rate changes. 
Protocol

Pretest

In the pretest, gas analyzers (Vmax Series 29C, Sensor Medics, CA, USA) were used to assess the energy consumption of the participants while they were resting and performing nonmaximal intensity exercises for precisely calculating the daily calorie consumption of each participant. After the participants arrived at the laboratory, they wore heart rate monitors (Polar, Finland), and their energy consumption was examined using gas analyzers. Next, they quietly rested for 20 minutes in the supine position, and their resting heart rate and energy consumption were recorded. They then performed nonmaximal intensity exercises for measuring their energy consumption during low-intensity activities. The participants stood on a treadmill with a slope of $0^{\circ}$ for 10 minutes to record their energy consumption during standing. The participants then walked or ran on the treadmill at five speeds, namely 1,2,3,4, and 5 miles per hour. The participants maintained each speed for 3 minutes for examining the relationship between the energy consumption and heart rate of the participants during low-intensity activities.

After the pretest, the participants were asked to perform their normal daily life activities while wearing the heart rate monitor; their heart rates were recorded for 24 hours. The 24-hour heart rate, the regression curve of the heart rate, and energy consumption calculated in the pretest were be used to calculate the total energy consumption of the participants. In the experiment, meals were adjusted so that the total calories of each meal met the 24-hour energy requirement of each participant. The method used to record energy consumption and the brand of heart rate monitors (Polar) used in this study have been described elsewhere $(12,20,21)$. Moreover, 24-hour energy consumption was recorded the day before the experiment, and the calories of meals were adjusted to meet the 24-hour energy requirement of each patient. The same methods were repeated on the fifth day of the experiment to confirm the 24-hour energy consumption changes of the two trials.

\section{Formal Experiment}

The experiment was conducted over 6 days. On the first day, the participants arrived at the laboratory at 08:00 and quietly rested for 20 minutes in the supine position; gas analyzers were used to record their energy consumption. Subsequently, the participants were randomly allocated to the time-restricted feeding trial or the control trial. The meals of the time-restricted feeding trial were provided at 12:00, 16:00, and 20:00, and the participants were required to consume all the food during this time. The meals of the control trial were provided at 08:00, 14:00, and 20:00, but the consumption time was not limited. In addition to regular meals, a snack with approximately 200 calories was provided to the participants for consumption. The participants in the time-restricted feeding trial were only allowed to consume the snack from 12:00 to 20:00, whereas no restrictions were imposed on the control trial for snack consumption. The meals of the participants were provided by dieticians. Based on the results of the pretest, the calories of each meal met the daily energy requirement of the participants. The macronutrient consumption for TRF and CON were listed in table 1. 
After experiment completion on the fifth day, the participants returned to the laboratory on the eighth day from 08:00 to 09:00. They rested for 10 minutes in the supine position, and gas analyzers were used to collect the gas data of the participants for 20 minutes. Next, a catheter was inserted into the forearm of each participant to collect fasting blood samples. After blood sample collection, the participants were provided with a specific high-fat meal. The participants rested quietly in the laboratory for 4 hours, and their blood lipid changes during this period were observed.

Oral fat tolerance test (OFTTQ

All oral fat tolerance test (OFTT) meals were designed and provided by dieticians, as previously described $(12,22,23)$. The meals included toast, butter, cheese, muesli, and cream. For every $\mathrm{kg}$ of the body weight of the participant, the meal provided $1.2 \mathrm{~g}$ of fat, $1.1 \mathrm{~g}$ of carbohydrate, $0.33 \mathrm{~g}$ of protein, and $16.5 \mathrm{kcal}$ of energy. The nutritional information was obtained from the nutritional facts on food packages. During the experiment, the participants were required to consume the OFTT meal within 15 minutes.

Blood collection

In the experiment, a catheter (Venflon 20G, Sweden) was inserted into the vein of the forearm, and a threeway stopcock (Connecta Ltd., Sweden) was used to collect $10 \mathrm{~mL}$ of blood each time. Blood was collected before meals, 30 minutes after meals, and every hour after meals up to the fourth hour. After each session of blood collection, $10 \mathrm{~mL}$ of isotonic saline water was used to clean the catheter to avoid blood clotting in the catheter.

The collected blood was immediately placed in blood collection tubes containing ethylenediaminetetraacetic acid. A cell counter was used to analyze the hematocrit (Sysmax KX-21N, Kobe, Japan). After the analysis, the blood was centrifuged for 20 minutes at $500 \times \mathrm{g}$ at $4{ }^{\circ} \mathrm{C}$. Blood plasma was obtained and was immediately placed in a $-80^{\circ} \mathrm{C}$ refrigerator for preservation and future biochemical analysis.

Blood biochemical analysis

TG, blood glucose, free fatty acid, and glycerol levels in blood were analyzed using an automated biochemistry analyzer (7020, Hitachi, Japan) and commercial reagents (GOD-PAP method, Randox, Ireland). The insulin concentration in blood plasma was analyzed using a chemiluminescence immunoassay analyzer (Elecsys 2010, Roche Diagnostics, Basel, Switzerland) and commercial reagents (Roche Diagnostics). The fat and carbohydrate oxidation rates were calculated using the following formula(24):

Fat oxidation $(\mathrm{g} / \mathrm{min})=1.695 \times \mathrm{VO} 2-1.701 \times \mathrm{VCO} 2$

Carbohydrate oxidation $(\mathrm{g} / \mathrm{min})=4.585 \times \mathrm{VCO} 2-3.226 \times \mathrm{VO} 2$

Statistical Analysis

Page 5/14 
All the data in this study are presented as average \pm standard deviation. First, the normality of the data was tested using the Shapiro-Wilk test. The fasting fat oxidation rate, blood biochemical values, and areas under the fat oxidation rate curve and the TG curve were analyzed using the paired sample $t$ test. The postprandial fat oxidation rate and blood biochemical values were analyzed using two-way ANOVA with repeated measures. If the data were significant, the Bonferroni method was used to perform post hoc comparisons. Using $\mathrm{G}^{*}$ power 3 , to achieve an alpha value of $5 \%$ and a power of 0.8 , the sample size of eight was considered sufficient for this study. The significance level was set ata $<0.05$.

\section{Result}

\section{Fasting plasma concentrations}

The fasting fat oxidation rate of the time-restricted feeding trial was significantly higher than that of the control trial $(p<0.001)$. The concentrations of TG, blood glucose, insulin, free fatty acids, and glycerol between the two trials were not significantly different $(p>0.05)$ (Table 1$)$.

Postprandial fat oxidation rate and area under the curve

The postprandial fat oxidation rate of the time-restricted feeding trial was significantly higher than that of the control trial (trial $\times$ time, $p=0.019$; trial, $p=0.004$; time, $p=0.256$; Figure $1 \mathrm{~A}$ ). The area under the postprandial fat oxidation rate curve of the time-restricted feeding trial was significantly larger than that of the control trial $(p=0.02$, Figure $1 \mathrm{~B})$.

Postprandial TG concentration and area under the curve

Postprandial TG concentrations were not significantly different between the time-restricted feeding and control trials (trial $\times$ time, $p=0.531$; trial, $p=0.850$; time, $p<0.001$; Figure $2 \mathrm{~A}$ ). The areas under the TG concentration curve were also not significantly different between the two trials ( $p=0.956$, Figure $2 \mathrm{~B}$ ).

\section{Postprandial blood biochemical indices}

The plasma glucose concentration was not significantly different between the two trials (trial $\times$ time, $p=$ 0.712; trial, $p=0.456$; time, $p=0.023$; Figure $3 \mathrm{~A}$ ). Figure 3B indicates that the insulin concentration was not significantly different between the trials (trial $\times$ time, $p=0.564$; trial, $p=0.558$; time, $p=0.027$; Figure $3 \mathrm{~B})$. Figure $3 \mathrm{C}$ indicates that the free fatty acid concentration was not significantly different between the trials (trial $\times$ time, $p=0.598$; trial, $p=0.976$; time, $p<0.001$; Figure $3 \mathrm{C}$ ). Figure $3 \mathrm{D}$ indicates that the glycerol concentration was not significantly different between the trials (trial $\times$ time, $p=0.925$; trial, $p=$ 0.064; time, $p<0.001$; Figure 3D).

\section{Discussion}

In this study, meals were provided that met the 24-hour energy requirement of the participants for 5 days, and the intervention was time-restricted feeding conducted at different parts of the day. The results 
revealed that time-restricted feeding effectively increased the fasting fat oxidation rate or the fat oxidation rate after the consumption of high-fat meals. However, the increased fat oxidation rate exerted no effects on the TG level following high-fat meals, 24-hour energy consumption, or reactions of blood biochemical substances.

This study confirmed that 5 days of time-restricted feeding effectively increased the fasting fat oxidation rate and the fat oxidation rate after the consumption of high-fat meals. Studies applying time-restricted feeding have mostly used interventions with a duration of a few weeks, and the results showed that timerestricted feeding decreased body weight and improved metabolism $(25,26)$. Studies that have utilized short-term time-restricted feeding have discovered that 4 days of early time-restricted feeding (consuming dinner before 15:00) effectively increased the fat oxidation rate and reduced appetite; however, it did not affect 24-hour energy consumption (18). A similar study demonstrated that 4 days of early time-restricted feeding improved the 24-hour blood glucose balance (19). In contrast to the aforementioned studies, this study used late time-restricted feeding (consuming dinner before 20:00). In addition, all the meals were prepared by the research team and were directly provided to the participants; hence, in this study, the diet of the participants could be more precisely controlled, instead of the participants consuming their own food. This study discovered that late time-restricted feeding produced results similar to those achieved by early time-restricted feeding. In addition, compared with the control trial, time-restricted feeding did not affect the 24-hour energy metabolism of the time-restricted feeding trial, and time-restricted feeding effectively reduced the fasting fat oxidation rate and the fat oxidation rate after the consumption of highfat meals. However, the glycerol and free fatty acid concentrations of the two trials were not different. Therefore, the exact mechanism through which time-restricted feeding increased the fat oxidation rate was unknown.

In this study, time-restricted feeding could effectively increase the fasting fat oxidation rate and the postprandial fat oxidation rate, but it did not affect the TG level after the consumption of high-fat meals. This result indicated that 5 days of short-term time-restricted feeding resulted in a shorter action time for the increase in the fat oxidation rate; thus, the postprandial TG level was not affected. Chiu et al. (2019) used three high-fat meals per day to change the fat oxidation rate of participants; although this method effectively increased the fat oxidation rate, it did not affect the TG level after the consumption of high-fat meals (14). This study demonstrated that the fat oxidation rate of the time-restricted feeding trial was significantly higher than that of the control trial; however, glycerol and free fatty acid concentrations were not significantly different. Therefore, although short-term time-restricted feeding effectively increased the fat oxidation rate, it did not affect the postprandial TG reaction.

Another possible reason for the intervention not affecting the TG level after the consumption of high-fat meals is that 5-day time-restricted feeding did not affect blood glucose and insulin concentrations. Studies have suggested that insulin sensitivity is a major factor that affects the TG level after the consumption of high-fat meals (27). Compared with late time-restricted feeding, early time-restricted feeding reduced postprandial blood glucose concentration to a higher extent in a previous study (28). However, that study did not limit the calorie intake, and participants were 55 years old and were at a high 
risk of diabetes. In comparison, this study provided all the meals to the participants during the experiment to ensure that the calorie intake of all the participants was equal. In addition, this study controlled the calorie intake to ensure that it met the 24-hour energy requirement of the participants, and the results revealed that fasting and postprandial blood glucose concentrations and the insulin concentration were unaffected. Accordingly, the insulin sensitivity of the participants remained unchanged; thus, the postprandial TG level was unaffected.

The main limitation of this study was the calculation of 24-hour energy consumption. The 24-hour energy consumption was determined through calculation, rather than through measurement by methods such as those using the respiratory chamber. Calculations would not be as accurate as actual measurements.

Studies have tested 24-hour energy consumption and yielded robust results using methods similar to that used in the present study $(12,20,21)$. Therefore, we believe that this method is still credible. In addition, this study did not collect data on the muscle glycogen or intramyocellular TG levels and did not analyze lipoprotein lipase activity. Therefore, this study could not explain the main reason for the nonsignificant effect of 5-day time-restricted feeding on postprandial TG.

\section{Conclusion}

This study discovered that consuming meals with the same amount of calories for 5 days and using time-restricted feeding as the intervention can effectively increase the fasting fat oxidation rate and the fat oxidation rate after the consumption of high-fat meals. However, the increased fat oxidation rate did not increase the TG level after the consumption of high-fat meals. Future studies can extend the duration of time-restricted feeding and can investigate how long-term time-restricted feeding affects the TG level after the consumption of high-fat meals.

\section{Declaration}

Ethics approval and consent to participate: This study was approved by the Institutional Review Board of Jen-Ai Hospital (110-10) in Taiwan.

\section{Consent for publication}

Not applicable.

\section{Availability of data and materials}

All relevant materials are presented in the present manuscript.

\section{Competing interests}

The authors declare that they have no competing interests. 
Funding: This study was funded by Ministry of Science and Technology in Taiwan (110-2410-H-028 -009).

Author Contributions: C.-H.C. (Chih-Hui Chiu) carried out the experiment, blood analysis and assisted the manuscript preparation. C.-H.C. (Che-Hsiu Chen) and M.-H.W. assisted the data analysis and manuscript preparation. Y.-C.H, Y.-Z.L, and B.-W.L assisted the experimental design, data analysis and manuscript preparation. All authors haveread and agreed to the published version of the manuscript.

\section{Acknowledgements}

Thanks for Sports Science Research Center of National Taiwan University of Sport to provide the equipment for this study.

\section{Authors' information}

${ }^{1}$ Graduate Program in Department of Exercise Health Science, National Taiwan University of Sport, Taichung 404, Taiwan. ${ }^{2}$ Department of Sport Performance, National Taiwan University of Sport, Taichung 404, Taiwan. ${ }^{3}$ Sport Recreation and Health Management Degree Program, Tunghai University, Taichung 404, Taiwan. ${ }^{4}$ Graduate Program in Department of Exercise Health Science, National Taiwan University of Sport, Taichung 404, Taiwan. ${ }^{5}$ Graduate Program in Department of Exercise Health Science, National Taiwan University of Sport, Taichung 404, Taiwan. ${ }^{6}$ Graduate Program in Department of Exercise Health Science, National Taiwan University of Sport, Taichung 404, Taiwan.

\section{References}

1. Liu H-H, Li J-J. Aging and dyslipidemia: a review of potential mechanisms. Ageing Research Reviews. 2015;19:43-52.

2. Nordestgaard BG, Benn M, Schnohr P, Tybjærg-Hansen A. Nonfasting triglycerides and risk of myocardial infarction, ischemic heart disease, and death in men and women. JAMA: The Journal of the American Medical Association. 2007;298(3):299-308.

3. Bansal S, Buring JE, Rifai N, Mora S, Sacks FM, Ridker PM. Fasting compared with nonfasting triglycerides and risk of cardiovascular events in women. JAMA: The Journal of the American Medical Association. 2007;298(3):309-16.

4. Langsted A, Freiberg J, Tybjærg-Hansen A, Schnohr P, Jensen GB, Nordestgaard B. Nonfasting cholesterol and triglycerides and association with risk of myocardial infarction and total mortality: the Copenhagen City Heart Study with 31 years of follow-up. Journal of Internal Medicine. 2011;270(1):6575 .

5. Ferreira AP, Ferreira CB, de Souza VC, de Almeida Córdova CO, Silva GCB, de Toledo Nóbrega O, et al. The influence of intense intermittent versus moderate continuous exercise on postprandial lipemia. 
Clinics. 2011;66(4):535.

6. Hurren NM, Eves FF, Blannin AK. Is the effect of prior exercise on postprandial lipaemia the same for a moderate-fat meal as it is for a high-fat meal? British Journal of Nutrition. 2011;105(4):506.

7. Miyashita M. Effects of continuous versus accumulated activity patterns on postprandial triacylglycerol concentrations in obese men. International Journal of Obesity. 2008;32(8):1271-8.

8. Miyashita M, Burns SF, Stensel DJ. Acute effects of accumulating exercise on postprandial lipemia and C-reactive protein concentrations in young men. International journal of sport nutrition and exercise metabolism. 2009;19(6):569.

9. Maraki MI, Sidossis LS. The latest on the effect of prior exercise on postprandial lipaemia. Sports Medicine. 2013;43(6):463-81.

10. Peddie MC, Rehrer NJ, Perry TL. Physical activity and postprandial lipidemia: are energy expenditure and lipoprotein lipase activity the real modulators of the positive effect? Progress in Lipid Research. 2012;51(1):11-22.

11. Trombold JR, Christmas KM, Machin DR, Kim I-Y, Coyle EF. Acute high-intensity endurance exercise is more effective than moderate-intensity exercise for attenuation of postprandial triglyceride elevation. Journal of Applied Physiology. 2013;114(6):792-800.

12. Yang T-J, Wu C-L, Chiu C-H. High-intensity intermittent exercise increases fat oxidation rate and reduces postprandial triglyceride concentrations. Nutrients. 2018;10(4):492.

13. Wilhelmsen A, Mallinson J, Jones R, Cooper S, Taylor T, Tsintzas K. Chronic effects of high-intensity interval training on postprandial lipemia in healthy men. Journal of Applied Physiology. 2019;127(6):1763-71.

14. Chiu C-H, Yang T-J, Chen C-H, Zeng M-J. High fat meals increases postprandial fat oxidation rate but not postprandial lipemia. Lipids in health and disease. 2019;18(1):1-7.

15. Sutton EF, Beyl R, Early KS, Cefalu WT, Ravussin E, Peterson CM. Early time-restricted feeding improves insulin sensitivity, blood pressure, and oxidative stress even without weight loss in men with prediabetes. Cell metabolism. 2018;27(6):1212-21. e3.

16. Liu B, Page AJ, Hutchison AT, Wittert GA, Heilbronn LK. Intermittent fasting increases energy expenditure and promotes adipose tissue browning in mice. Nutrition. 2019;66:38-43.

17. Liu B, Page AJ, Hatzinikolas G, Chen M, Wittert GA, Heilbronn LK. Intermittent fasting improves glucose tolerance and promotes adipose tissue remodeling in male mice fed a high-fat diet. Endocrinology. 2019;160(1):169-80. 
18. Ravussin E, Beyl RA, Poggiogalle E, Hsia DS, Peterson CM. Early time-restricted feeding reduces appetite and increases fat oxidation but does not affect energy expenditure in humans. Obesity. 2019;27(8):1244-54.

19. Jamshed H, Beyl RA, Della Manna DL, Yang ES, Ravussin E, Peterson CM. Early time-restricted feeding improves 24-hour glucose levels and affects markers of the circadian clock, aging, and autophagy in humans. Nutrients. 2019;11(6):1234.

20. Silva AM, Santos DA, Matias CN, Judice PB, Magalhães J, Ekelund U, et al. Accuracy of a combined heart rate and motion sensor for assessing energy expenditure in free-living adults during a double-blind crossover caffeine trial using doubly labeled water as the reference method. European journal of clinical nutrition. 2015;69(1):20-7.

21. Santos DA, Silva AM, Matias CN, Magalhães JP, Fields DA, Minderico CS, et al. Validity of a combined heart rate and motion sensor for the measurement of free-living energy expenditure in very active individuals. Journal of Science and Medicine in Sport. 2014;17(4):387-93.

22. Chiu C-H, Burns SF, Yang T-J, Chang Y-H, Chen Y-L, Chang C-K, et al. Energy replacement using glucose does not increase postprandial lipemia after moderate intensity exercise. Lipids in health and disease. 2014;13(1):177.

23. Chiu C-H, Yang T-J, Liang HJ, Chang C-K, Wu C-L. A Single Bout of Exercise Reduces Postprandial Lipemia but Has No Delayed Effect on Hemorheological Variables. The Chinese journal of physiology. 2018;61(3):181-7.

24. Frayn K. Calculation of substrate oxidation rates in vivo from gaseous exchange. Journal of applied physiology. 1983;55(2):628-34.

25. Pellegrini M, Cioffi I, Evangelista A, Ponzo V, Goitre I, Ciccone G, et al. Effects of time-restricted feeding on body weight and metabolism. A systematic review and meta-analysis. Reviews in Endocrine and Metabolic Disorders. 2020;21(1):17-33.

26. Gabel K, Hoddy KK, Haggerty N, Song J, Kroeger CM, Trepanowski JF, et al. Effects of 8-hour time restricted feeding on body weight and metabolic disease risk factors in obese adults: A pilot study. Nutrition and healthy aging. 2018;4(4):345-53.

27. Guerci B, Verges B, Durlach V, Hadjadj S, Drouin P, Paul J. Relationship between altered postprandial lipemia and insulin resistance in normolipidemic and normoglucose tolerant obese patients. International journal of obesity. 2000;24(4):468-78.

28. Hutchison AT, Regmi P, Manoogian EN, Fleischer JG, Wittert GA, Panda S, et al. Time-restricted feeding improves glucose tolerance in men at risk for type 2 diabetes: a randomized crossover trial. Obesity. 2019;27(5):724-32. 


\section{Tables}

Table 1. The macronutrient consumption for TRF and CON

\begin{tabular}{|llll|}
\hline & TRF & CON & P value \\
\hline Carbohydrate $(\mathrm{g})$ & $340.4 \pm 28.0$ & $342.2 \pm 31.4$ & 0.904 \\
\hline Protein $(\mathrm{g})$ & $124.7 \pm 11.3$ & $128.7 \pm 31.4$ & 0.599 \\
\hline Fat $(\mathrm{g})$ & $74.9 \pm 7.9$ & $72.1 \pm 10.2$ & 0.552 \\
\hline Total calorie (Kcal) & $2533.6 \pm 240.9$ & $2532.0 \pm 272.0$ & 0.983 \\
\hline
\end{tabular}

Values are mean $\mathrm{SD}, \mathrm{n}=8$. TRF, time-restricted feeding trial; $\mathrm{CON}$, control trial.

Table 2. The participants physiological information and fasting plasma biochemistry.

\begin{tabular}{|llll|}
\hline & TRF & CON & p value \\
\hline 24 h energy expenditure $($ Kcal) & $2541.5 \pm 1406.2$ & $2388.6 \pm 1370.4$ & 0.419 \\
\hline TG $(\mathrm{mmol} / \mathrm{L})$ & $1.16 \pm 0.4$ & $1.45 \pm 0.7$ & 0.077 \\
\hline Glucose $(\mathrm{mmol} / \mathrm{L})$ & $70.6 \pm 5.3$ & $71.0 \pm 3.2$ & 0.793 \\
\hline Insulin $(\mathrm{pmol} / \mathrm{L})$ & $7.8 \pm 3.2$ & $7.6 \pm 4.5$ & 0.931 \\
\hline NEFA $(\mathrm{mmol} / \mathrm{L})$ & $0.38 \pm 017$ & $0.35 \pm 0.08$ & 0.655 \\
\hline Glycerol $(\mu \mathrm{mol} / \mathrm{L})$ & $33.4 \pm 17.0$ & $27.9 \pm 10.5$ & 0.190 \\
\hline Fat oxidation $(\mathrm{g} / \mathrm{min})$ & $0.14 \pm 0.05$ & $0.04 \pm 0.03$ & $<0.001$ \\
\hline Carbohydrate oxidation $(\mathrm{g} / \mathrm{min})$ & $0.21 \pm 0.12$ & $0.26 \pm 0.14$ & 0.167 \\
\hline
\end{tabular}

Values are mean $S D, n=8$. TRF, time-restricted feeding trial; CON, control trial; TG, triglyceride; NEFA, Nonesterified fatty acids.

\section{Figures}



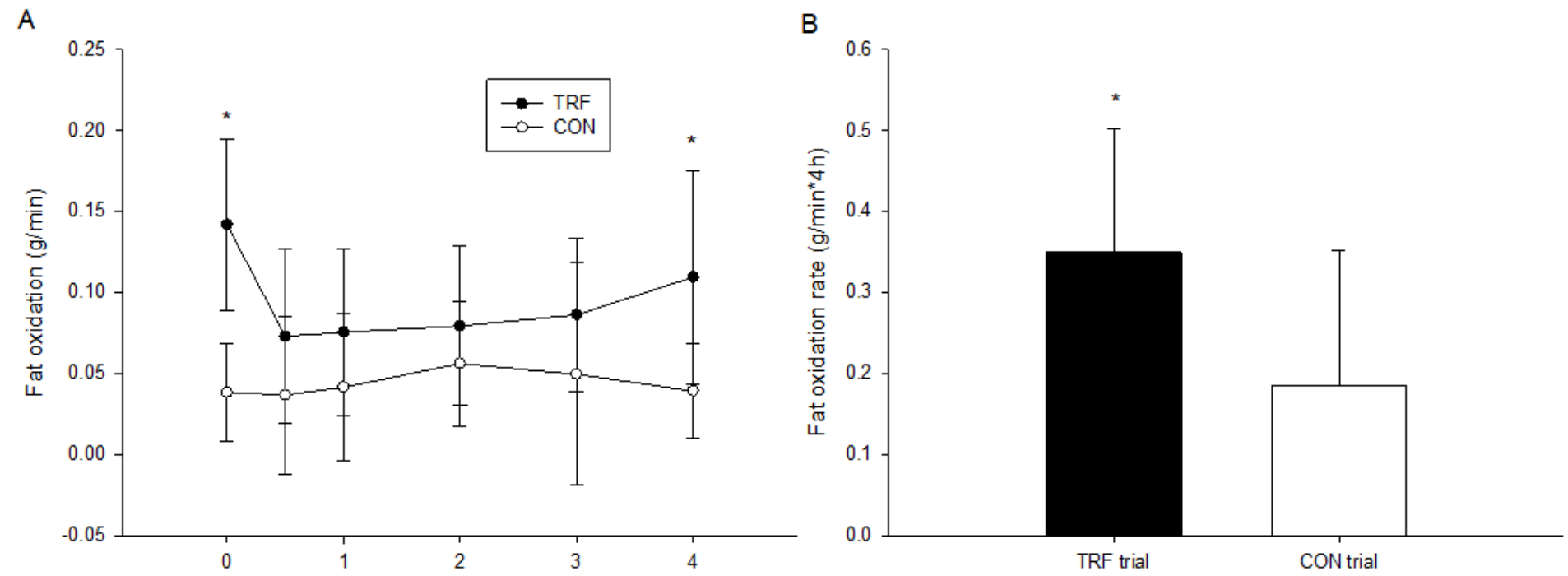

Figure 1

The postprandial fat oxidation over the 4 hours $(A)$ and the fat oxidation rate area under the curve in 4 hours (B). ${ }^{*}$ TRF was significantly higher than those for the CON.
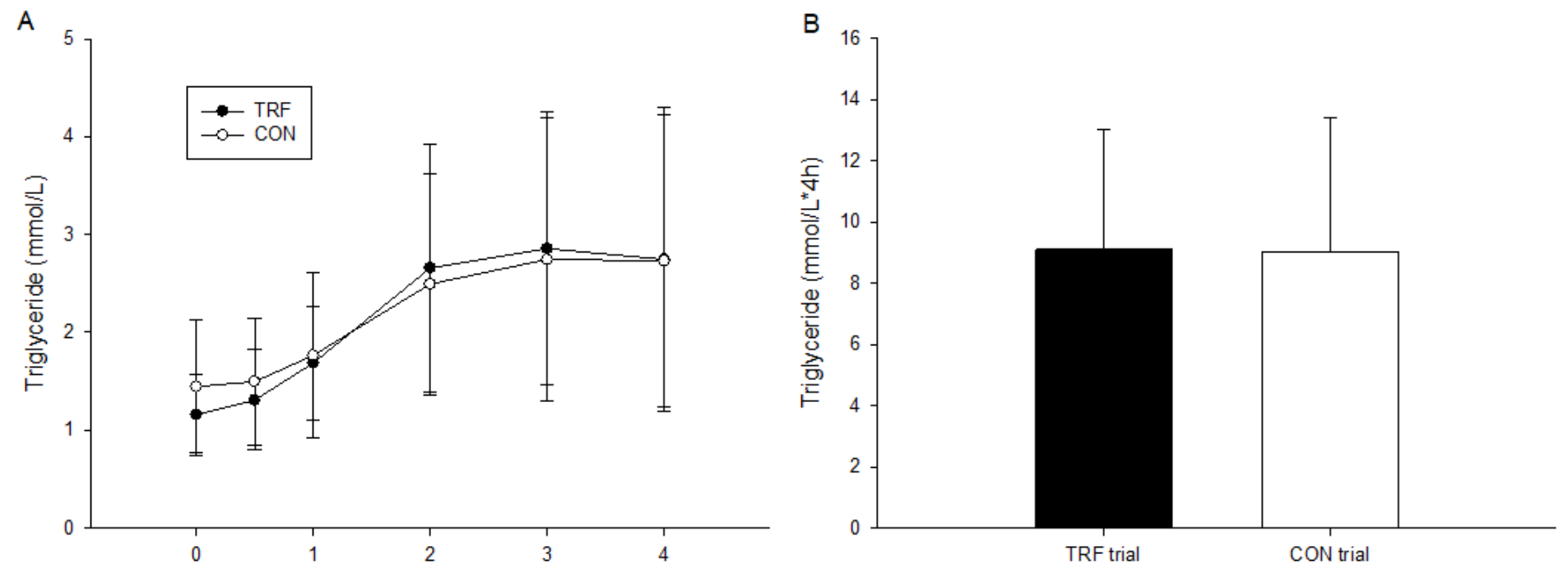

Figure 2

The postprandial triglycerides concentrations over the 4 hours $(A)$ and the TG area under the curve in 4 hours (B). ${ }^{*}$ mean TRF was significantly higher than those for the CON. 

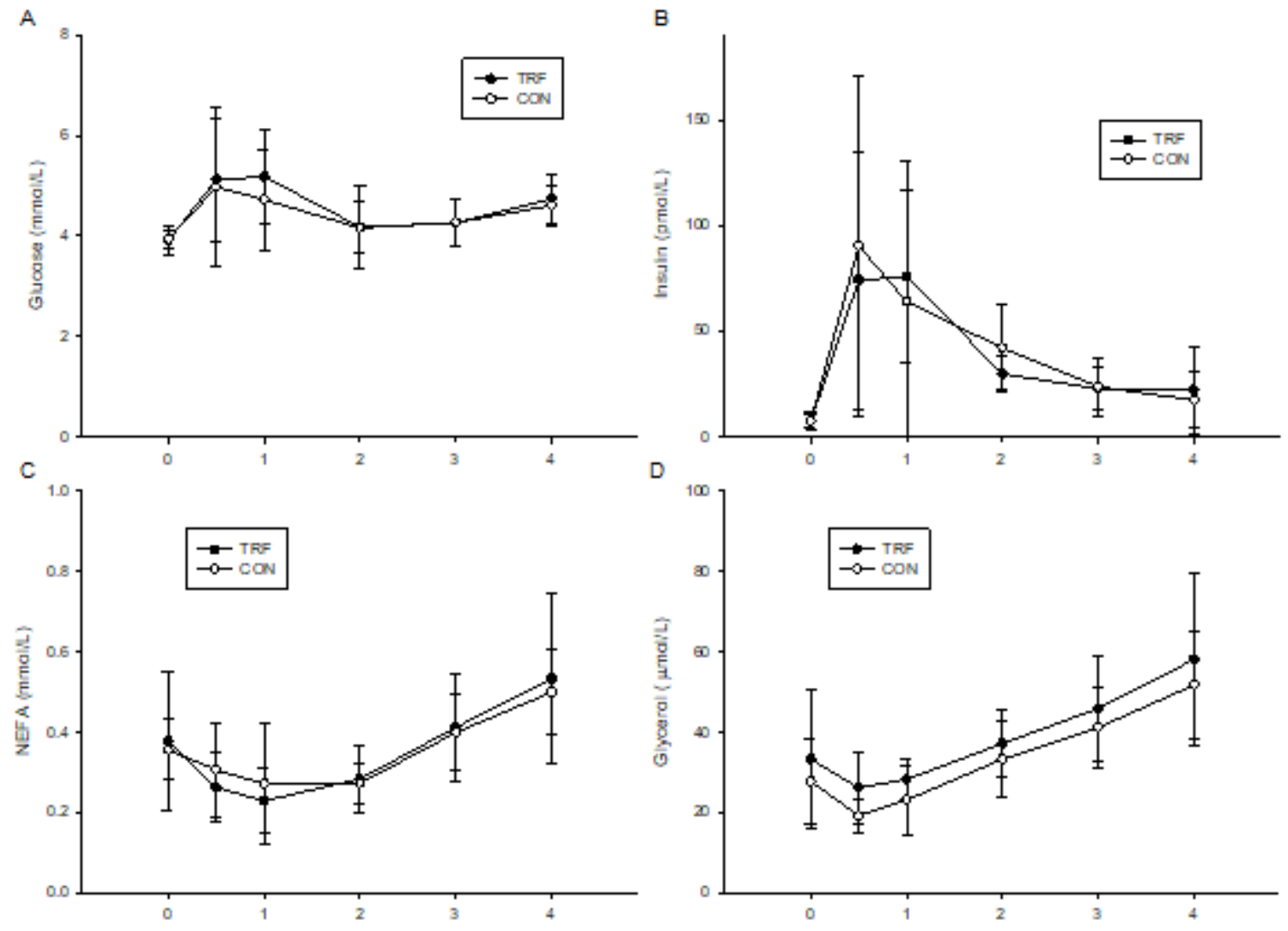

Figure 3

The postprandial glucose concentrations over the 4 hours (A), insulin concentrations over the 4 hours (B), glycerol concentrations over the 4 hours (C) and non-esterified fatty acids concentrations over the 4 hours. 